\title{
Subjetividad y aprendizaje del inglés: experiencias vividas desde un programa de desarrollo profesional
}

\section{Subjectivity and learning English: experiences lived from a professional development program}

QUINTERO, Sonia R. ${ }^{1}$

QUICENO, Evelin C. ${ }^{2}$

URIBE, Claudia M. ${ }^{3}$

JARAMILLO, Bairon ${ }^{4}$

\section{Resumen}

El artículo que se presenta a continuación, se deriva del proyecto de investigación "Impacto del Programa Desarrollo Profesional Docente Pre K-English en las Prácticas de Aula en Educación Inicial de las Maestras Participantes" desarrollado por un equipo de investigadores adscritos a la Facultad de Educación y Humanidades de la Universidad Católica Luis Amigó durante 2018. Este ejercicio se realizó con la pretensión de determinar las percepciones de las maestras de educación inicial frente al proceso de aprendizaje del inglés. Para ello se diseñaron instrumentos como el cuestionario, la entrevista, las observaciones y el grupo focal. Los resultados de este ejercicio investigativo conducen al fortalecimiento de las comunidades académicas de los maestros de educación infantil y la enseñanza del inglés como lengua extranjera.

Palabras clave: subjetividad, aprendizaje, inglés, educación

\begin{abstract}
This article is derived from the research project "Impact of the Pre-K-English Teacher Professional Development Program on Classroom Practices in Early Childhood Education of Participating Teachers" developed by a team of researchers ascribed to the Faculty of Education and Humanities from Luis Amigó Catholic University during 2018. This exercise aimed to determine the perceptions of the teachers of initial education regarding the process of learning English. For its development the research team devised instruments for data collection, among which are the questionnaire, the interview, the observations and the focus group, The results of this research exercise lead to the strengthening of the academic communities of early childhood education teachers and the teaching of English as a foreign language.

key words: education, english, learning, subjectivity.
\end{abstract}

\footnotetext{
${ }^{1}$ Sonia Ruth Quintero Arrubla: Psicóloga, Licenciada en Pedagogía Reeducativa, Especialista en Docencia Investigativa Universitaria, Magister en Educación y docente Universidad Católica Luis Amigó. Correo electrónico sonia.quinteroar@amigo.edu.co

2 Evelin Cristina Quiceno Sossa: Licenciada en Lenguas Extranjeras con énfasis en inglés, Licenciada en Educación Preescolar, Magíster en procesos de enseñanza aprendizaje y enseñanza de segundas lenguas énfasis inglés y docente Universidad Católica Luis Amigó. Correo electrónico evelin.quicenoso@amigo.edu.co

${ }^{3}$ Claudia María Uribe Hoyos: Licenciada en Lenguas Extranjeras con énfasis en inglés, Especialista en Docencia Universitaria y docente Universidad Católica Luis Amigó. Correo electrónico claudia.uribeho@amigo.edu.co

${ }^{4}$ Bairon Jaramillo Valencia: Licenciada en Lenguas Extranjeras con énfasis en inglés, Magíster en Educación, doctorando en Educación y docente Universidad Católica Luis Amigó. Correo electrónico

bairon.jaramillova@amigo.edu.co
} 


\section{Introducción}

El presente artículo se deriva del proyecto de investigación "Impacto del Programa Desarrollo Profesional Docente Pre-K English en las Prácticas de Aula en Educación Inicial de las Maestras Participantes" desarrollado por un grupo de investigadores adscritos a la Facultad de Educación y Humanidades de la Universidad Católica Luis Amigó durante 2018. Este ejercicio se basa en los principios de la investigación cualitativa haciendo uso de instrumentos mixtos como son el cuestionario, las observaciones y el grupo focal.

El proyecto investigativo tiene como objetivo general, analizar el impacto del Programa Desarrollo Profesional Docente Pre- K English en las prácticas de aula en educación inicial de las maestras participantes de las instituciones educativas del sector público del municipio de Medellín y dentro de los objetivos específicos se contempla, el determinar las percepciones de las maestras frente a la enseñanza del inglés y su efecto en las prácticas pedagógicas y didácticas de las mismas.

Pre-K English se concibe el inglés como una lengua que cohabita de manera natural con el español en las aulas de educación inicial de las maestras participantes del proyecto, lo cual lleva a entender que el inglés debe dejar de ser una simple lista de conceptos, actividades o clase aislada y convertirse en una lengua que represente los sentires, las vivencias y las creencias de la comunidad educativa -maestras y estudiantes. En palabras de Maturana \& Uribe, (2017)

Partiendo de la premisa de que tener ambos idiomas, español e inglés, coexistiendo el aula de educación inicial favorece la participación de los participantes en el Programa y su disposición hacia el lenguaje y la deconstrucción de sus prácticas docentes, el Pre-K Desarrollo profesional de maestros de inglés, el programa se incluyó en el ámbito de tres Principios pedagógicos, didácticos y teóricos. (pág. 167)

En el año 2015, en Colombia se expidió la Ley 1753, denominada Ley del Plan Nacional de Desarrollo 2014-2018 "Todos por un nuevo país" la cual, entre otros aspectos da especial relevancia a la educación. Este plan se convierte en la ruta que debe orientar el conjunto de acciones que permitan cumplir las metas en materia de educación. Es así como desde el propósito nacional "Colombia la más educada en el 2025" se determinan las líneas estratégicas de la política educativa del Ministerio de Educación Nacional, en la que se resalta lo siguiente:

Para materializar el propósito de hacer de Colombia el país mejor educado de América Latina en el 2025, el Ministerio de Educación Nacional planteó las líneas estratégicas que determinarán el rumbo de la educación para los próximos años: Excelencia Docente, Jornada Única, Colombia Bilingüe, Colombia Libre de Analfabetismo y Más Acceso a la Educación Superior de Calidad. (Ministerio de Educación Nacional [MEN], 2015, p.2)

Como puede verse, Colombia bilingüe, dentro de un plan de desarrollo deriva lineamientos que se traducen en tareas para el sector educativo, concretamente en los planes y proyectos que se contienen en los (Proyectos Educativos Institucionales para ser operativizados por los maestros en cada institución. Sin embargo, la pregunta que se genera es si éstos están preparados para asumir el reto Colombia Bilingüe, teniendo en cuenta que la labor educativa va más allá de un simple desarrollo de funciones y tareas emanadas de la política pública.

En este orden de ideas, el desarrollo de la competencia comunicativa en inglés como lengua extranjera debe fortalecerse desde la educación inicial y convocar a la formación de los maestros en el marco de la integralidad; es decir, no solo enfatizando en la incorporación de la lengua como sistema, sino en la revisión de aspectos pedagógicos y didácticos que las lleven a interrogarse frente a la manera como conducen los procesos educativos. 
Pensar en el aprendizaje de una lengua extranjera por parte de los maestros para enseñarla en las aulas de clase, permite plantear un segundo interrogante: ¿aprender inglés está en el deseo de las maestras de educación infantil? Un cuestionamiento necesario al momento de pensar en el reto Colombia Bilingüe, pues uno de los protagonistas es el maestro que está llamado a conectar su deseo con la realización de la tarea; sin embargo, este es un asunto que se complejiza a la hora de llevarlo a la práctica.

Hoy día, la comunidad académica está abocada a plantear reflexiones en torno al mejoramiento de las propuestas educativas y la calidad de la educación en nuestro medio, desde la generación de espacios de análisis, discusión y proposición sobre el saber específico, su enseñabilidad y sobre los procesos de enseñanza aprendizaje. Es por ello que este proyecto investigativo busca determinar la percepción de las maestras al tener que verse como aprendices de la lengua para desarrollar la competencia comunicativa en inglés y llevar este idioma a las aulas de educación infantil de manera natural y en contexto, con el propósito de establecer reflexiones en torno a las necesidades de formación profesional docente y sus implicaciones en los procesos de enseñanza aprendizaje, en este caso del inglés como lengua extranjera. Tal y como lo señalan Maturana \& Uribe, (2017) en su artículo: Promoción de la transformación personal y profesional de docentes de preescolar mediante la articulación de dos programas de extensión universitaria: una reflexión.

Esta experiencia pedagógica demostró que los programas de extensión deberían abarcar un papel más activo en la construcción de la sociedad, teniendo en cuenta sus necesidades ofreciendo programas centrados en el crecimiento social en lugar de simplemente cursos de formación. (pág. 175)

\section{Metodología}

El ejercicio investigativo descrito en este documento se desarrolla desde un enfoque cualitativo con una postura socio-crítica por su intención de comprender las limitaciones que envolvían a los participantes y el ofrecer comprensiones que les permitieran trascender dichas limitaciones (Porta, L \& Silva, M. 2003). La población participante de esta investigación constaba de 150 maestras que habían pertenecido al Programa Desarrollo Profesional Docente Pre K-English ejecutado durante el periodo 2014-2017 y que respondían a los siguientes criterios: (a) haberse graduado del programa; (b) laborar como maestras de educación inicial y (c) participar voluntariamente del ejercicio investigativo. El proceso se desarrolló en tres fases: la primera, el planteamiento y formulación; la segunda, la recolección de la información; y la tercera, dedicada al análisis, conclusiones e implicaciones generadas desde la indagación. Para recoger los datos se diseñaron instrumentos tales como el cuestionario, la entrevista, el grupo focal y la observación de manera tal que se logrará un proceso confiable y riguroso.

\section{Resultados}

Desde el objetivo que plantea revisar las percepciones de las maestras frente a la enseñanza del inglés y su efecto en el trabajo educativo con los niños de educación preescolar, se tienen los siguientes resultados:

En esta investigación se evidencia que, en una población de 150 maestras a su ingreso al programa Pre-K English, el $9 \%$ responde que aprender inglés es sencillo, mientras que el $91 \%$ considera que aprender inglés es muy difícil, siendo esta una consideración que sostienen y defienden. Con esta información, los investigadores se dan a la tarea de indagar por las razones de esta percepción, encontrándose que, de manera generalizada las maestras relacionan aspectos que tiene que ver con situaciones vividas en su trayectoria escolar con el grupo de compañeros y con los maestros en los niveles de educación básica, en donde se implicaba la función de autoridad de sus maestros de inglés y la actitud asumida por los grupos escolares, además de referir aspectos desfavorables relacionados con la didáctica de la enseñanza del inglés como lengua extranjera. 
Figura 1

Percepciones de las maestras frente al aprendizaje del inglés antes de su ingreso al Programa de Desarrollo profesional Docente Pre-K English, 2014. (Datos recolectados por los investigadores).

Aprender inglés es sencillo

Aprender inglés es muy dificil

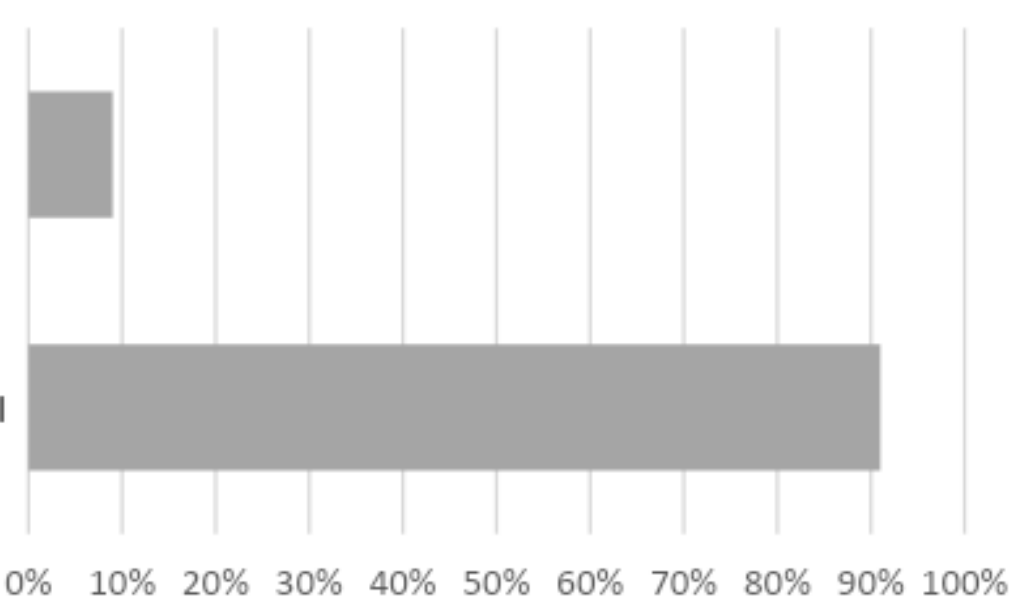

Al revisar las percepciones de las maestras y las razones que aducen, se encuentra que, es este un asunto que invita a pensar en la enorme responsabilidad del maestro en los procesos educativos, desde la consideración de que lo que se implica en las aulas de clase, va más allá del encuentro con el deseo de saber, lo que se pone en evidencia, es la subjetividad humana que va a determinar la relación de cada sujeto consigo mismo, con el otro y con lo otro.

Es importante partir del concepto de subjetividad, para luego situarlo en el escenario educativo y dar lugar a las reflexiones que se generan desde el estudio de caso. En este orden de ideas, se parte de lo planteado por Cubides (como se citó en Díaz, 2005) quien define la subjetividad, como "un proceso por el cual uno mismo y sus acciones se convierten en el objeto de observación, a partir de examinar y comprender cómo las narrativas estructuran las propias experiencias y cómo nuestras experiencias estructuran nuestras narrativas" (párr. 2). Permitiendo con ello ubicar la subjetividad en el campo de las narrativas, es decir la subjetividad como un hecho del lenguaje, derivado de las vivencias de cada persona.

De otro lado, González (2008) frente a la subjetividad en su artículo Subjetividad social, sujeto y representaciones sociales, dice:

El sentido subjetivo está asociado de forma inseparable a las configuraciones subjetivas de la subjetividad individual; en consecuencia, no se puede analizar sólo de forma puntual en el curso de la expresión del lenguaje. El sentido subjetivo expresa las producciones simbólicas y emocionales, configuradas en las dimensiones histórica y social de las actividades humanas; sin embargo, éstas no expresan apenas el momento actual de un sistema de relaciones, sino la historia, tanto de las personas implicadas en un espacio social, como de ese espacio social en su articulación con otros. De esa forma, el sentido subjetivo fundamenta una definición de subjetividad, que no se restringe a los procesos y a las formas de organización de la subjetividad individual, sino que implica la definición de una subjetividad social. (p.37)

La subjetividad es concebida como conciencia específicamente cultural e histórica, tal y como lo enuncian Jiménez y Ramírez (2015) cuando la definen como "un devenir permanente a través de configuraciones de sentidos y, por consiguiente, experiencias" (p.84). 
La subjetividad entonces, es asumida como un hecho del lenguaje y el lenguaje es entendido como el conjunto de códigos que permiten la expresión y la interacción con los otros en la vía de comunicarse. Vista así, la subjetividad aparece como un proceso que configura la integralidad de cada persona en el marco de sus experiencias de vida, en las que son determinantes el afecto, el límite, los valores y las interacciones. Por consiguiente, como lo plantea Ramírez y Anzaldúa (2014) "La subjetividad se conforma a partir de la relación de los sujetos con la naturaleza, la autoridad y el gobierno de sí mismos" (p. 176).

Para este análisis, se hace necesario ubicar el concepto de subjetividad en el contexto educativo, para referir dos aspectos básicamente, el primero el lugar de autoridad del maestro y el segundo, la manera como orienta sus afectos en la relación transferencial que tiene lugar en el aula de clase.

La función del maestro, en tanto encargo social, reviste un lugar de autoridad, de representación simbólica de ley. Es una figura central en el aula de clase, y su función desencadena afectos conscientes e inconscientes en los estudiantes, un asunto que bien pueden generar barreras en el proceso educativo, pero también posibilidades para el encuentro con el deseo de saber y conocer. En el capítulo, autoridad y nuevo orden, del texto ¿Sin Límite? José Fernando Velásquez (2007) dice que, la autoridad es una función que tiene un representante y busca un orden social. Por consiguiente, pensar en la autoridad de un maestro implica su historia, implica su propia subjetividad. En este mismo sentido, respecto a la autoridad Hebe Tizio (2006) menciona lo siguiente:

La autoridad ha cambiado, decimos, pero eso no es una catástrofe, solo se trata de ver qué modelo de autoridad conviene para este tiempo. Sobre esto sabemos que han caducado varios modelos pero que los límites se necesitan. La idea de límite es para posibilitar: no a esto, pero sí a esto otro. Hay que saber que tanto el autoritarismo como el dejar hacer sin límite son las dos caras de lo peor, es decir, de un funcionamiento superyoico. (p. 36)

El lugar de autoridad que desempeña el docente, desencadena de manera inconsciente sentimientos que los estudiantes le transfieren de sus historias de vida, aplicándose la relación con el conocimiento. En su artículo, la transferencia en el proceso educativo, Ramírez (2011), plantea frente a la formación de los docentes en relación con la transferencia en el proceso educativo lo siguiente, en términos de llamado a los maestros:

El tercer llamado es a formar un adulto significativo y firme, que ayude a los estudiantes a comprender su sentido de vida y reconozca las potencialidades de los sujetos (pero también sus limitaciones) desde una actitud abierta y tranquila, basada en la confianza y el respeto mutuo, que posibilite de esta manera la instalación del amor de transferencia, en primer momento de carácter imaginario, es decir, una identificación del niño posicionado como yo ideal frente al maestro, para dar paso luego a la transferencia simbólica, en este caso a la identificación con el deseo de saber. (p. 87)

La manera como el maestro se posicione frente a la autoridad, puede interferir en el deseo de saber y precisamente en esta investigación se encuentra que, al ingreso de las maestras en el programa de desarrollo profesional docente Pre-K English, las resistencias frente al aprendizaje de una lengua extranjera, en este caso al inglés, eran generalizadas y, estaban estrechamente asociadas a las vivencias en un aula de clase, en donde la relación que estas tuvieron con sus maestros de inglés en la educación básica estuvo marcado por la descalificación y la hostilidad; especialmente al evidenciar falencias en la pronunciación, la escritura y la comprensión del inglés. Posiciones verticales en el ejercicio de la autoridad de los maestros referido como un estilo inflexible, tradicional y autocrático que menguaba la participación y generaba resistencias del lado de los estudiantes en el encuentro con la lengua extranjera. 
El estilo de autoridad autocrática de los maestros en las clases de inglés que eran impartidas al conjunto de maestras participantes de Pre-k English, en sus historias académicas en básica primaria, las lleva a la creación de imaginarios, al afloramiento de emociones y sentimientos que se convirtieron en barreras y temores que las llevaron a predisponerse con el aprendizaje de la lengua. Frente a esta situación, en las entrevistas, ellas manifiestan sentirse expuestas ante un grupo que contempla sus falencias complejiza la situación; por ello en muchas ocasiones, recurrieron a la indisciplina y la desatención con el desarrollo de la clase, como mecanismos de defensa para evitar ser miradas y evaluadas por el grupo en general.

En esta investigación, resulta particular darse cuenta de que ante lo penoso que les resultara en el pasado el encuentro con el aprendizaje de la lengua extranjera inglés, las maestras participantes de Pre-k English afirman que con el grupo de compañeros de básica empezaron a expresan sus percepciones frente a aprender inglés; afirman que es difícil, es decir lo ponen en el lenguaje, lo difunden, lo repiten y lo determinan como verdad, una verdad que sostienen a lo largo de sus historias de vida académica: 'aprender inglés es muy difícil'.

Al respecto de lo anterior, al entrevistar a una de las maestras y preguntarle por su percepción frente a aprender inglés antes de su llegada al programa de desarrollo profesional docente plantea:

Aprender inglés siempre fue muy duro para mí, todo el tiempo creí que nunca podría asimilar ese conocimiento y hoy que analizo, me doy cuenta de que estuvo relacionado con la manera en que la profesora nos enseñaba, era muy duro ponerse en frente de los compañeros para ser mirado y descalificado, eso no se soporta, y en inglés sabes o no sabes, no hay otra posibilidad...ella miraba fijo, hablaba con su tono de voz aguda y regañón, yo miraba cómo se enojaba cuando no sabíamos y eso era demoledor....odiaba esa clase, odiaba ese verbo to be, creo que yo hasta me enfermaba cuando había clase de inglés (Maestra entrevistada. Comunicación personal, mayo de 2018).

Como puede notarse la influencia que tiene un maestro sobre un grupo y específicamente sobre un estudiante, tiene dimensiones impensables. La manera como éste se dirija a los estudiantes y el contenido de sus palabras, puede ser un decreto que se copia y se carga durante toda la vida. En este mismo sentido, otra maestra opina frente al interrogante:

Aprender inglés nunca fue fácil y a uno lo predisponen los compañeros y uno termina creyéndolo, y pienso que tiene mucho que ver con la maestra o el maestro que enseña, la actitud, la confianza que transmita, la manera como corrija, las oportunidades que dé a los estudiantes, las estrategias...Personalmente me fue muy mal con el inglés, siempre lo vi imposible y las resistencias que se generaron en mi frente al inglés son sencillamente sorprendentes, menos mal estamos en otro momento (Maestra entrevistada. Comunicación personal, mayo de 2018).

Del lado del maestro, no siempre hay una pregunta por sí mismo y por los efectos de su intervención pedagógica, pues es mucho más sencillo pensar en los faltantes que tiene un grupo o un estudiante en particular, que cuestionarse no solo frente a la didáctica que implementa, sino frente a su actitud como maestro, una actitud que implica todo su ser, pues no se puede olvidar que el maestro también tiene una historia que lo ha determinado, por consiguiente está cargado de subjetividad y transfiere afectos de manera inconsciente. El maestro desencadena contra transferencias hacia el estudiante o hacia el grupo y genera también barreras en la relación con el otro en el proceso educativo.

Con lo dicho, se hace necesario hacer mención al concepto de transferencia, para ubicarla en el proceso educativo, y de esta forma, considerar sus efectos en la relación maestro estudiante. Se parte entonces, de este concepto definido desde el psicoanálisis de la siguiente manera: la transferencia, se define en psicoanálisis, como "el proceso en virtud del cual los deseos inconscientes se actualizan sobre ciertos objetos, dentro de un 
determinado tipo de relación establecida con ellos. Se trata de una repetición de prototipos infantiles, vivida con un marcado sentimiento de actualidad" (Laplanche y Pontalis, 1996, p. 459).

Hablar de transferencia afectiva implica además remitirse al proceso edípico y la importancia de su resolución, puesto que es ahí cuando cada persona queda instalada en el afecto y en el límite de manera particular, una manera que determina un proceso de construcción de la propia subjetividad humana. En correspondencia, Quintero, Ramírez y Jaramillo (2015) plantean lo siguiente:

El proceso edípico es el origen de los fenómenos transferenciales que luego van a ser su emergencia en las instituciones educativas a partir de las relaciones que el niño establece con la maestra, con los pares, con los grupos y con el mundo exterior. (p.3)

Este planteamiento lleva a pensar que, cuando un maestro no tiene una pregunta por sí mismo y por los efectos de sus actitudes, de sus comportamientos en la institución con relación al grupo o a cada uno de los sujetos que lo conforman, será arduo que comprenda que en muchas ocasiones el comportamiento de un estudiante puede ser una manifestación de un malestar que emerge de la relación transferencial.

En este análisis es claro que las actitudes del maestro, desde el lugar de autoridad y afecto, pueden desencadenar barreras con el aprendizaje, en este caso, del inglés como lengua extranjera. Cuando un estudiante se siente valioso en un grupo y muy especialmente considera que, el maestro cree en él y en el desarrollo de sus capacidades, se cree estimulado, animado y dispuesto para cuestionar, buscar, resolver y avanzar, siendo este elemento un motivador importante en su proceso, no solo de aprendizaje, sino de construcción de su ser en el mundo. De ahí lo determinante que es que, un maestro esté reconciliado con su ser y con su historia, para que pueda orientar sus afectos, desde una pregunta por lo que es, lo que expresa y lo que hace en la relación con sus estudiantes.

En esta investigación se pudo constatar que fue tan fuerte la resistencia generada en las maestras cuando fueron estudiantes de básica que, al paso de los años, para algunas resultaba irreconciliable su relación con el inglés en los procesos de aprendizaje, al punto que, en el rol de maestras en las instituciones educativas con los niños de educación infantil, solo el $15 \%$ manifiesta en el momento del ingreso al programa, que enseñaban inglés. Es decir, su percepción genera resistencias, obstaculizando la enseñanza en los grupos de educación infantil.

Al respecto, Arredondo et al. (como se citó en McBride, 2009) refieren en el artículo, las percepciones estudiantiles sobre las técnicas utilizadas en la enseñanza del inglés como lengua extranjera, lo siguiente:

Es esencial que se sepa algo de las percepciones que tienen los estudiantes en cuanto a las prácticas que han experimentado en sus clases de segundas lenguas. Las creencias de los estudiantes sobre el proceso de aprendizaje pueden afectar a su motivación, a su receptividad con respecto a las actividades en clase, a sus estrategias de estudio, y, a la larga, a su rendimiento. (p.98).

La autora plantea que en el estudio que realiza, encuentra una diferencia entre creencias y percepciones de los estudiantes según el idioma que estudiaban; además, expone que esta situación estaba relacionada con las expectativas hacia el uso del idioma en el futuro y en las oportunidades para usarlo. Sin embargo, para el caso de las maestras del programa de desarrollo profesional docente Pre-K English, el asunto de las resistencias fue tan fuerte que supera cualquier expectativa frente al uso del inglés.

En este orden de ideas, Maturana (2011) en su artículo, La Enseñanza del Inglés en Tiempos del Plan Nacional de Bilingüismo en algunas Instituciones Públicas: Factores Lingüísticos y Pedagógicos, frente a la competencia comunicativa, en el factor lingüístico dice que, "se encontró que la autoimagen y las creencias subyacen al concepto de competencia comunicativa de las maestras como elementos que la determinan y atraviesan" (p.75). 
En tanto afirma que las educadoras construyen su propio ego lingüístico partiendo de la percepción que tengan de la competencia comunicativa en inglés. Y si se piensa en el caso de las maestras participantes de esta investigación, a su ingreso a Pre-K English, su ego lingüístico podría estar afectado por las experiencias negativas de su historial académico en la básica.

Desde el programa Pre-K English se pudo establecer un proceso de formación profesional que parte de elementos esenciales: (a) la relación tutor estudiante, enmarcada en el respeto y la confianza para dar seguridad; (b) la generación de ambientes de aprendizaje participativos llenos de sentido y significado en el marco de la atención a la diversidad desde el enfoque de la educación inclusiva; (c) la transversalización del inglés en todo el proceso de enseñanza aprendizaje, lo cual implica que no se enseña desde una clase específica, sino que hace parte de la cotidianidad del aula (e), la generación de una cultura de la participación desde estrategias pedagógicas que incluyen los lenguajes expresivos o actividades rectoras; y (f) la evaluación en el aula como proceso formativo y sistémico.

Al revisar si el programa de desarrollo profesional docente Pre-K English logra un cambio significativo en la percepción de las maestras frente al aprendizaje del inglés se encuentra el siguiente resultado:

Figura 2

Percepciones de las maestras frente al aprendizaje del inglés luego de su ingreso al Programa de Desarrollo profesional Docente Pre-K English, 2018. (Datos recolectados por los investigadores).

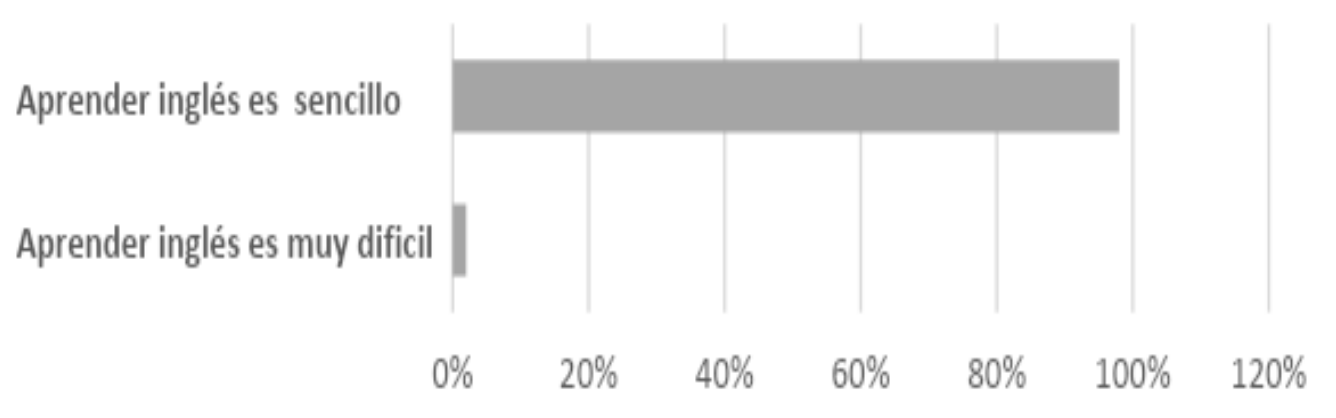

Tal y como se muestra, el programa de desarrollo profesional docente Pre-K English permite el derrumbamiento de barreras frente al aprendizaje del inglés. Es así cómo se logra pasar del $91 \%$ en un grupo de 150 de una percepción de "aprender inglés es muy difícil" a un $2 \%$, en correspondencia. Las maestras hoy, luego de su paso por el programa, cambiaron su percepción, logrando articular lo aprendido y desaprendido en su experiencia educativa con los niños, es decir, logran impactar la didáctica del aula.

En uno de los grupos focales realizados para este ejercicio investigativo, una de las maestras frente a la percepción que tiene hoy, luego de su paso por el programa de desarrollo profesional docente Pre-K English, manifiesta:

Hoy recuerdo cuando llegué al programa, temerosa y muy ansiosa, pensando en lo difícil que era aprender inglés, pero al encontrar ese ambiente de confianza y seguridad, pensé que sería posible, aún recuerdo cuando hablamos de los imaginarios y de las experiencias en la básica, eso fue determinante y sentí que no era la única...que además nos estaban ofreciendo un programa no solo de capacitación para aprender una lengua, la propuesta fue muy acertada desde el programa pues aquí tocamos también el ser, la didáctica y por eso hoy enseñar inglés a mis niños es lo más gratificante en mi experiencia como profesional de la educación. (Maestra participante del proyecto 
de desarrollo profesional docente Pre-K English en el periodo 2015-2017. Comunicación personal, junio de 2018).

Otra de ellas en el grupo focal expresa:

Estoy muy cuestionada luego de mi salida de Pre-K, hoy pienso en los docentes que tuve y en lo que pasó con el inglés en mi vida...pienso en la enorme responsabilidad de la actitud con los niños, pienso en cómo nuestras acciones desde el lugar de maestros sí afectan la historia de vida, pero con satisfacción también veo cómo es posible generar ambientes en los que la didáctica incluya a todos y provoque participación y deseo de aprender......mi percepción cambió y también mi manera de enseñar inglés, hoy disfruto enseñar y ese que ellos disfrutan aprender y no les niego que hasta los padres aprenden (Maestra participante del proyecto de desarrollo profesional docente Pre-K English en el periodo 2015-2017. Comunicación personal, junio de 2018).

Como se dijo anteriormente, al ingreso al programa Pre-K-English, solo el 15\% en un grupo de 150 maestras dijo enseñar inglés a los niños dentro de la jornada académica. Lo hacían dedicando el tiempo a dos temas específicos: los números y los colores. Hoy, luego del paso por el programa el $100 \%$ afirma enseñar inglés de manera transversal y replicando las estrategias que se implementaron; es así como el juego, el cuento, las canciones, la poesía, las composiciones, el dibujo y muchos otros lenguajes expresivos son los protagonistas en las instituciones educativas para enriquecer en los niños los aspectos lingüísticos y el disfrute de aprender inglés.

La experiencia de formación profesional docente vivida, por las maestras en Pre-K English, les permitió la revisión de la didáctica en el aula de clase en su rol como maestras de educación infantil. En este sentido, también se pudo apreciar que las docentes, antes de su paso por este programa, no orientaban sus estrategias hacia el aprendizaje del inglés como una lengua que pertenecía al aula, en atención a que no estaba contemplado en el plan del nivel, por consiguiente, no lo consideraban necesario y esta creencia, sumada a su posición personal frente al aprendizaje del inglés, hace que ni siquiera se considerara como fundamental en el proceso formativo de los niños de educación inicial.

\section{Conclusiones}

Este ejercicio de investigación devela que el programa de desarrollo profesional docente Pre-K English permite a las maestras, en tanto estudiantes, el derrumbamiento de barreras frente al aprendizaje del inglés. Es así cómo se logra pasar de la percepción "aprender inglés es difícil" de $91 \%$ en un grupo de 150 a un 2\%; lo que demuestra, que hay una notable transformación en las maestras en su posición subjetiva frente al aprendizaje del inglés. Lo anterior llevó a la comprensión de su situación y estimuló la participación y el disfrute de lo vivido en el programa; aspecto que, a la vez, les posibilita desde su rol de maestras en los preescolares del municipio de Medellín, enseñar inglés a los niños de manera transversal, generando ambientes cargados de sentido y significado.

Un asunto determinante en la didáctica es la relación maestro-estudiante, en la cual tiene lugar un proceso de comunicación marcada por aspectos objetivos y subjetivos, en este sentido, cobra importancia el lugar del maestro como figura de autoridad y, por consiguiente, desencadenante de afectos inconscientes que posibilitan o dificultan el deseo de aprender. En los procesos de enseñanza y aprendizaje del inglés como lengua extranjera debe considerarse la subjetividad humana, desde las percepciones que se construyen a lo largo de la experiencia académica, pues de esta manera se derrumban imaginarios, resistencias y barreras que llevan al aprendizaje de otra lengua como parte integral del desarrollo humano. 
Cuando se piensa en la formación de maestros para la enseñanza del inglés como lengua extranjera y se trasciende del hecho de capacitar al proponer un programa de desarrollo profesional docente se hace evidente la integralidad de los miembros de la comunidad académica, como aprendices y maestros. Por eso es crucial que se creen programas, a nivel local, regional, y nacional que sean de participación voluntaria, con ejes específicos que respondan a las necesidades reales de los participantes y cuya puesta en escena contribuyan a las políticas públicas sin dejar de lado la voz y la experticia de las comunidades académicas. (Maturana \& Uribe)

\section{Referencias Bibliográficas}

Díaz, A. (2005). Subjetividad política y ciudadanía juvenil. Les cahiers psychologie politique, (7). http://lodel.irevues.inist.fr/cahierspsychologiepolitique/index.php?id=1140

González, F. (agosto, 2008). Subjetividad social, sujeto y representaciones sociales. Diversitas, 4 (2), 225-243. Centro Universitario de Brasilia. http://pepsic.bvsalud.org/scielo.php?script=sci_arttext\&pid=S179499982008000200002

Jiménez, K., y Ramírez. V. (septiembre, 2016). Aproximaciones a la subjetividad y socialización política de jóvenes excombatientes del conflicto armado en Colombia. Trabajo Social, (18), 77-90. Universidad Nacional de Colombia. https://revistas.unal.edu.co/index.php/tsocial/article/view/58573

Laplanche, J., y Pontalis, J. (1996). Diccionario de Psicoanálisis. Buenos Aires, Argentina: Paidós.

Maturana, L. (noviembre, 2011). La enseñanza del inglés en tiempos del Plan Nacional de Bilingüismo en algunas instituciones públicas: factores lingüísticos y pedagógicos. Colombian Applied Linguistics Journal, 13(2), 74-87. http://www.scielo.org.co/pdf/calj/v13n2/v13n2a06.pdf

Maturana-Patarroyo, L. M. Uribe-Hoyos, C. M. (2018). Enhancing Pre-K Teachers' Personal and Professional Transformation by Articulating Two University Extension Approaches: A Reflection. Íkala, Revista de Lenguaje y Cultura, 23(1), 161-176.

DOI: https://doi.org/10.17533/udea.ikala.v23n01a11

McBride, K. (agosto, 2009). Percepciones estudiantiles sobre las técnicas utilizadas en la enseñanza del inglés como lengua extranjera. Universum, 24 (2), 94-112. Universidad de Talca. http://dx.doi.org/10.4067/S0718-23762009000200006

Ministerio de Educación Nacional de Colombia. (2015). Colombia, la mejor educada en el 2025. Líneas estratégicas de la política educativa del Ministerio de Educación Nacional. https://www.mineducacion.gov.co/1621/articles-355154_foto_portada.pdf

Porta, L. \& Silva, M. (2003) "La investigación cualitativa: El Análisis de Contenido en la investigación educativa”. Dr. LUIS PORTA - Universidad Nacional de Mar del Plata. Mag. MIRIAM SILVA - Universidad Nacional de la Patagonia Austral

Quintero, S., Ramírez, L., y Jaramillo, B. (2015). Los procesos grupales y la convivencia en el preescolar. Simposio Internacional de Educación, Pedagogía y Currículo. Chile.

Quintero Quintero, M., Giraldo, J. L. (2006). Sujeto y educación: hacia una ética del acto educativo. Aula abierta.

Ramírez, B., y Anzaldúa, R. (diciembre, 2014). Subjetividad y socialización en la era digital. Argumentos, 27 (76), 171-189. UAM-Xochimilco. http://www.scielo.org.mx/scielo.php?script=sci_arttext\&pid=S018757952014000300009 
Ramírez, L. (enero, 2011). La transferencia en el proceso educativo. Revista Colombiana de Ciencias Sociales, 2 (1), 85-89. Universidad Católica Luis Amigó.

http://www.funlam.edu.co/revistas/index.php/RCCS/article/view/27

Sierra, G. (octubre de 2007). ¿Sin Límite? El extravío de la autoridad y su efecto en niños y adolescentes. Corporación Ser especial. Colombia.

Tizio, E. (2006). La educación y los cuerpos de hoy. Freudiana.

Universidad Católica Luis Amigó (2013). Estrategia de formación para maestros de Preescolar.[White paper]. Licenciatura en Inglés y Licenciatura en Preescolar. FUNLAM.

\footnotetext{
Esta obra está bajo una Licencia Creative Commons Attribución-NoCommercial 4.0 International

(cc) BY-NC
} 